\title{
Conduta Obstétrica no Óbito Fetal
}

\author{
Obstetrical Management of Fetal Death
}

Márcia M A de Aquino, Ana Cláudia Guedes, Maria Rita S Mesquita Mirie Hernandez, José Guilherme Cecatti

\section{RESUM0}

Apesar de 80 a 90\% dos fetos mortos poderem ser eliminados espontaneamente após duas a três semanas do óbito, a indução do parto tem sido a conduta mais utilizada. $O$ objetivo deste estudo foi avaliar os resultados da indução de parto em casos de óbito fetal intra-útero com idade gestacional a partir de 20 semanas. Foi um estudo clínico descritivo realizado no Hospital Maternidade Leonor Mendes de Barros, em São Paulo. Foram analisadas 122 gestantes com esse diagnóstico quanto às características sociodemográficas, causas de óbito fetal, antecedentes obstétricos e características do parto (forma de indução, via de parto, complicações). Os procedimentos estatísticos utilizados foram cálculo da média e desviopadrão e $\chi^{2}$. As principais causas identificadas de morte fetal foram hipertensão arterial $e$ infecções. A droga mais utilizada para a indução do parto foi o misoprostol (37,7\%), seguido da ocitocina (19,7\%). Em 27\% dos casos o trabalho de parto iniciou espontaneamente. $O$ tempo médio de indução foi de 3 horas. A maior parte teve parto vaginal e em 9, $1 \%$ a cesárea foi realizada. Concluiu-se que a indução de parto de feto morto é segura e eficaz, independentemente do método utilizado. O misoprostol, utilizado por via vaginal, é especialmente útil nos casos de colo desfavorável, por seu efeito modificador sobre ele.

PALAVRAS-CHAVE: Óbito fetal. Ocitocina. Prostaglandina. Resolução da gravidez. Trabalho de parto.

\section{Introdução}

Historicamente a conduta obstétrica na gestante com feto morto modificou-se graças aos progressos propedêuticos e terapêuticos ${ }^{3,4}$. A perda fetal representa um episódio dos mais frustrantes e de difícil elaboração na vida de uma mulher, além de representar o insucesso na atividade profissional

Hospital Maternidade Leonor Mendes de Barros, São Paulo, SP, Departamento de Tocoginecologia CAISM/FCM/UNICAMP Correspondência:

José Guilherme Cecatti

Caixa Postal 6030

13081-970 Campinas SP

Fax: 019-2393687 do médico obstetra. Essa situação geradora de conflitos impede que a antiga e tradicional conduta de se esperar pelo desencadeamento espontâneo do trabalho de parto seja ainda a opção preferencial de qualquer das duas partes envolvidas. $\mathrm{Na}$ atualidade há uma tendência de conduta ativa em razão do quadro de ansiedade que, em geral, essas mulheres apresentam e também pela possibilidade da ocorrência de coagulação intravascular disseminada quando há retenção do feto morto por período superior a quatro semanas ${ }^{6}$.

Muitos métodos de indução já foram propostos e utilizados em todo o mundo. Entre eles, incluem-se a introdução de substâncias hipertônicas na câmara âmnica, a utilização de corpos estranhos extra-amnióticos como sondas ou 
laminárias, o uso de ocitocina e de prostaglandinas ${ }^{2,5,7,10,11}$

O uso das substâncias hipertônicas foi bastante difundido nas décadas de 60 e 70, porém foi abandonado em razão da grande morbidade que apresentava $^{2,11}$. A utilização de laminárias não pode ser considerada um procedimento rotineiro no Brasil, já que o produto não é comercializado e poucos serviços têm alguma experiência com esse método $^{5,8}$. Já a utilização de sondas colocadas extra-amnioticamente para o preparo do colo, previamente à indução, é um procedimento relativamente antigo que vem sendo resgatado, porém, preferencialmente para a indução de gestações de termo com feto vivo, considerando que já existem atualmente melhores opções em termos de eficácia, quando a vitalidade do feto não representa mais um parâmetro a ser vigilado ${ }^{7}$.

Quanto à ocitocina, seu uso para indução do trabalho de parto em gestações com idade gestacional inferior a 28 semanas, ou com colo uterino desfavorável, tem mostrado baixa eficácia. Já as prostaglandinas têm a propriedade de promover um amadurecimento do colo uterino que parece estar relacionado com um aumento da atividade da colagenase que promove amolecimento e apagamento da cérvix uterina e também a capacidade de induzir a atividade uterina contrátil em todas as fases da gestação ${ }^{4,9}$. No Brasil, entretanto, nunca de dispôs de prostaglandina $\mathrm{E}_{2}$ para uso em Obstetrícia, o que equivale a dizer que nunca se dispôs de um agente eficaz no preparo do colo uterino previamente à indução do trabalho de parto.

O lançamento no mercado brasileiro, em 1986, do misoprostol, produto sintético análogo à prostaglandina $\mathrm{E}_{1}$ para tratamento da úlcera péptica, levou alguns obstetras a testá-lo em casos de morte fetal anteparto com colo uterino desfavorável à indução com ocitocina, com resultados bastante favoráveis ${ }^{3,4,8}$, além de apresentar menor custo e maior estabilidade farmacodinâmica em relação às demais prostaglandinas.

Alguns estudos recentes têm mostrado a boa eficácia do misoprostol para a indução do parto de feto morto, com baixa incidência de efeitos colaterais, principalmente quando administrado por via vaginal. No entanto, ainda é necessário o estabelecimento da menor dose útil ${ }^{4,8}$.

Com esse propósito, o objetivo deste estudo foi avaliar os resultados da indução de parto em casos de óbito fetal intra-útero, utilizando-se diferentes esquemas de indução.

\section{Material e Métodos}

Este foi um estudo clínico descritivo onde foram avaliadas 122 gestantes com diagnóstico de óbito fetal anteparto e idade gestacional igual ou superior a 20 semanas, internadas no Hospital Maternidade Leonor Mendes de Barros para resolução do caso, no período de agosto de 1996 a maio de 1997. Os métodos utilizados para o amadurecimento do colo uterino e/ou indução do parto foram: misoprostol via vaginal ou oral, ocitocina endovenosa ou a associação de ambos (não simultaneamente), escolhidos pelo médico encarregado do caso por critérios clínicos nãopadronizados.

Para a coleta dos dados utilizou-se um formulário onde constam informações sobre as características sociodemográficas e gestacionais das mulheres, condições cervicais prévias à indução, droga utilizada, via de parto, dados do natimorto e intercorrências durante a indução ou parto. Esses instrumentos foram revisados quanto à qualidade e legitimidade das informações, e os dados codificados e armazenados em arquivo de computador, após testes de consistência. Para análise dos dados foram utilizados os procedimentos estatísticos de cálculo de média e desvio-padrão, além do $\chi^{2}$, por meio do pacote estatístico EPI.INFO 6.02.

\section{Resultados}

As gestantes com óbito fetal, incluídas nessa casuística, apresentaram média de idade em torno de 27 anos, tinham tido 4 consultas pré-natal, eram em média tercigestas e tinham idade gestacional média de 28 semanas. Detalhes sobre as características dessas mulheres estão contidos em outra publicação ${ }^{1}$. Em relação ao peso fetal, observou-se que cerca de dois terços dos recémnascidos apresentaram peso igual ou inferior a 1500 g (Tabela 1). Cerca de $12 \%$ dos natimortos pesavam menos de $500 \mathrm{~g}$, mas os casos foram incluídos no estudo porque havia uma confirmação ecográfica prévia de idade gestacional de 20 semanas ou mais. Somente $15 \%$ dos casos tiveram identificada uma causa certa do óbito. As mais prevalentes foram a hipertensão arterial, seguida das várias causas infecciosas ${ }^{1}$.

Dos métodos de indução, o misoprostol foi o mais utilizado, seguido pela ocitocina e pela associação de ambos (Tabela 2). Quanto à via de parto, predominou a via vaginal em cerca de $92 \%$ das vezes, tendo a cesárea sido utilizada somente 
em 11 casos (Tabela 3), sendo dez dessas por iteratividade e uma por falha de indução, visto que mais de $8 \%$ dos casos tinha duas ou mais cesáreas anteriores (Tabela 4).

$\mathrm{Na}$ avaliação da associação entre o método de indução e o tipo de parto, observou-se que todos os casos nos quais o misoprostol foi utilizado isoladamente evoluíram para parto normal, ao passo que cerca de $4 \%$ dos casos em que se utilizou somente ocitocina houve necessidade de realização

Tabela 1 - Distribuição percentual das gestantes com óbito fetal, segundo o peso do RN.

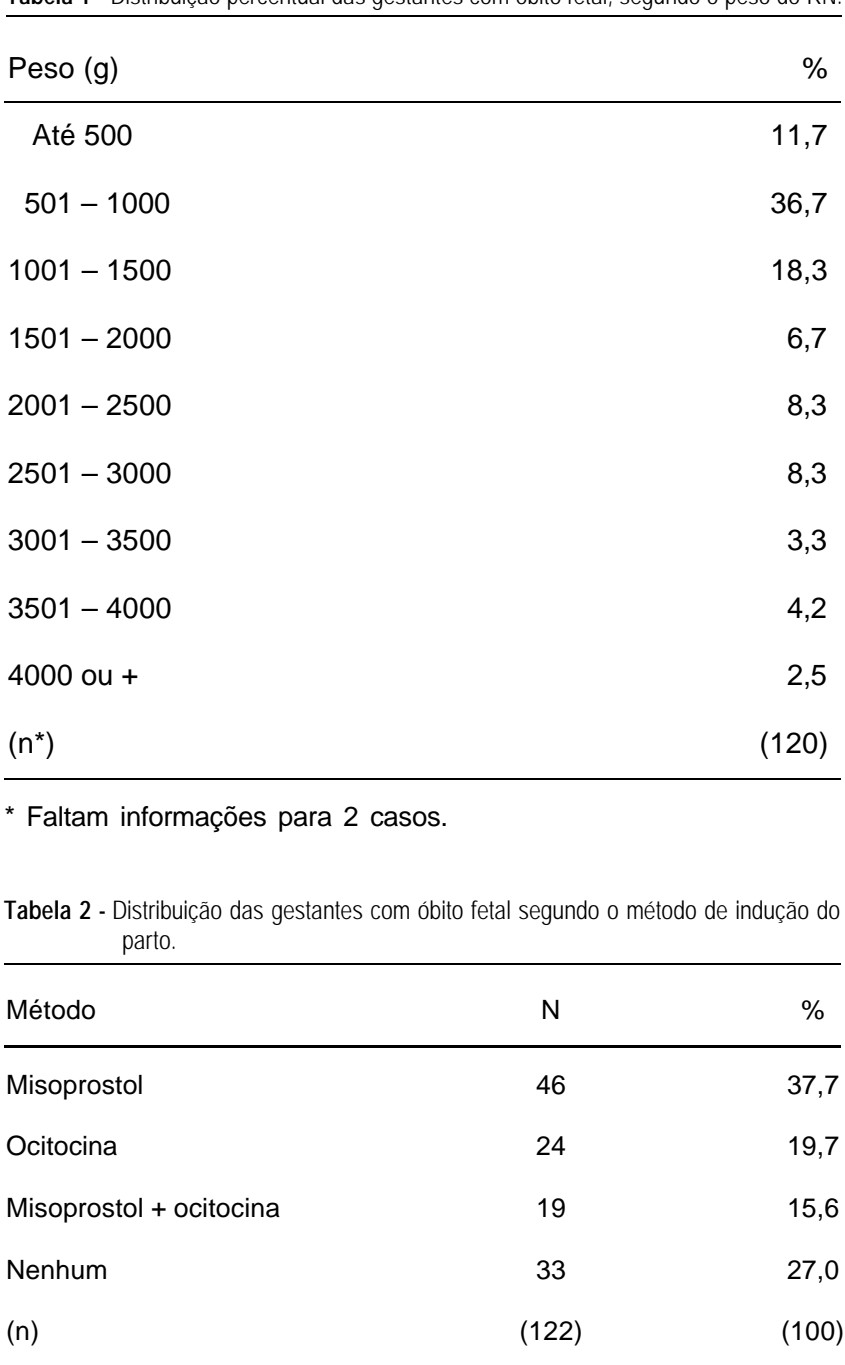

Tabela 3 - Distribuição das gestantes com óbito fetal segundo o tipo de parto.

\begin{tabular}{lcc}
\hline Tipo de parto & $\mathrm{N}$ & $\%$ \\
\hline Parto normal & 110 & 90,1 \\
Parto fórcipe & 01 & 0,8 \\
Parto cesárea & 11 & 9,1 \\
(n) & $(122)$ & $(100)$ \\
\hline
\end{tabular}

de cesárea (Tabela 5).

O misoprostol foi utilizado na dose total mínima de $50 \mu \mathrm{g}$ e na dose total máxima de $1600 \mu \mathrm{g}$, sendo a dose total média utilizada de $340 \mu \mathrm{g}$. O tempo médio de indução foi quase duas vezes maior nos casos em que o misoprostol foi utilizado isoladamente, do que quando somente a ocitocina foi utilizada (Tabela 6).

Observou-se que as características do colo uterino quanto à posição, grau de esvaecimento e dilatação eram mais favoráveis à indução nos casos em que somente a ocitocina foi utilizada em comparação aos casos em que somente o misoprostol foi ministrado. Essa diferença foi estatisticamente significante, entretanto, somente para o grau de esvaecimento do colo (Tabela 7).

Quanto às intercorrências durante a indução e complicações no parto, observou-se que, embora o número de casos tenha sido maior no grupo da ocitocina, não houve diferença estatisticamente significativa entre eles (Tabela 8).

Tabela 4 - Distribuição das gestantes com óbito fetal segundo o número de cesáreas anteriores.

\begin{tabular}{lcc}
\hline № de cesáreas & $\mathrm{N}$ & $\%$ \\
Zero & 93 & 76,2 \\
01 & 19 & 15,6 \\
02 & 08 & 6,6 \\
03 & 02 & 1,6 \\
(n) & $(122)$ & $(100)$
\end{tabular}

Tabela 5 - Distribuição percentual das gestantes com óbito fetal por método de indução segundo 0 tipo de parto.

\begin{tabular}{lcccc}
\hline Tipo de parto & Nenhum & Ocitocina & Misoprostol & Misoprostol + Ocitocina \\
\hline Parto normal & 75,8 & 91,8 & 100 & 89,5 \\
Parto fórcipe & - & 4,1 & - & - \\
Parto cesárea & 24,2 & 4,1 & - & 10,5 \\
(n) & $(33)$ & $(24)$ & $(46)$ & (19) \\
\hline
\end{tabular}

Tabela 6 - Tempo de indução (em horas) das gestantes com óbito fetal de acordo com 0 método de indução.

\begin{tabular}{lcc}
\hline Tempo & Misoprostol & Ocitocina \\
\hline Média & 14,3 & 8,3 \\
Min & 4,0 & 1,0 \\
Max & 96,0 & 24,0 \\
DP & 14,5 & 6,9 \\
\hline
\end{tabular}


Tabela 7 - Distribuição das gestantes com óbito fetal por método de indução segundo as condições cervicais.

\begin{tabular}{|c|c|c|}
\hline Colo do útero & Ocitocina & Misoprostol \\
\hline \multicolumn{3}{|l|}{ Posição } \\
\hline Medianizado & 43,5 & 23,9 \\
\hline Posteriorizado & 56,5 & 76,1 \\
\hline (n) & (23) & $(46)$ \\
\hline$\chi^{2}=2,77 \quad p=0,0096 \quad \mathrm{NS}$ & & \\
\hline \multicolumn{3}{|l|}{ Esvaecimento } \\
\hline Médio & 47,8 & 15,2 \\
\hline Grosso & 52,2 & 84,8 \\
\hline (n) & (23) & $(46)$ \\
\hline$\chi^{2}=6,85 \quad p=0,008$ & & \\
\hline \multicolumn{3}{|l|}{ Dilatação } \\
\hline Impérvio & 33,3 & 41,3 \\
\hline Dilatação até $1,5 \mathrm{~cm}$ & 16,6 & 34,8 \\
\hline Dilatação 2,0 cm & 12,5 & 8,7 \\
\hline Dilatação $\geq 3,0$ cm & 37,6 & 15,2 \\
\hline (n) & (24) & $(46)$ \\
\hline$p=0,125 \quad N S$ & & \\
\hline
\end{tabular}

Tabela 8 - Distribuição percentual das gestantes com óbito fetal por método de indução segundo a presença de intercorrências na indução ou complicações no parto

\begin{tabular}{|c|c|c|c|c|}
\hline & & & Ocitocina & Misoprostol \\
\hline \multirow{4}{*}{ Intercorrências } & \multicolumn{2}{|c|}{ Sim } & 6,5 & 8,3 \\
\hline & \multicolumn{2}{|c|}{ Não } & 93,5 & 91,7 \\
\hline & \multicolumn{2}{|c|}{$(\mathrm{N})$} & (46) & (24) \\
\hline & $\chi^{2}=0,04$ & $p=0,83$ & & \\
\hline \multirow{4}{*}{ Complicações } & \multicolumn{2}{|c|}{ Sim } & 19,6 & 20,8 \\
\hline & \multicolumn{2}{|c|}{ Não } & 80,4 & 79,2 \\
\hline & \multicolumn{2}{|c|}{$(\mathrm{N})$} & (46) & (24) \\
\hline & $\chi^{2}=0,04$ & $p=0,85$ & & \\
\hline
\end{tabular}

\section{Discussão}

Os resultados obtidos neste estudo mostram que a indução do parto de feto morto é segura e eficaz, independentemente do método utilizado, corroborando a tendência mais atual de conduta ativa com a finalidade de diminuir os quadros de ansiedade materna e distúrbios da coagulação sangüinea ${ }^{3,8}$.

Pode ainda mostrar que o misoprostol é especialmente útil nos casos de colo desfavorável, por seu efeito modificador sobre ele. No entanto, não houve aleatoriedade na escolha dos métodos; as mulheres foram selecionadas para o uso de determinado método de acordo com a idade gestacional e condições cervicais, por isso não se fez comparações através de testes de significância estatística entre os grupos, por motivos óbvios.

Os métodos de indução utilizados foram a ocitocina endovenosa, o misoprostol preferencialmente por via vaginal, ou misoprostol seguido por ocitocina (não simultaneamente). O critério de escolha para o misoprostol foi o colo desfavorável para indução e/ou idade gestacional inferior a 28 semanas. Nos casos em que o misoprostol promoveu um amadurecimento do colo, mas não houve expulsão do feto morto por não se ter desencadeado o trabalho de parto, indicou-se ainda o uso complementar da ocitocina.

A ocitocina como método de primeira escolha, portanto, foi utilizada em casos mais favoráveis, conforme se observa quando se avaliam as mulheres segundo as condições cervicais: a maior parte $(84,8 \%)$ das mulheres que teve o trabalho de parto induzido pelo misoprostol apresentava um colo uterino sem esvaecimento, ao passo que essa condição estava presente em cerca de apenas metade dos casos em que a ocitocina foi utilizada para aquele fim.

Quanto à via de parto, observou-se que os casos mais desfavoráveis à indução, que necessitaram do misoprostol e da ocitocina tiveram indicação de cesárea em cerca de $10,0 \%$ e no grupo da ocitocina em 4,0\%. No grupo tratado com misoprostol, que apresentava colo desfavorável, mas com idade gestacional menor, não houve indicação de cesárea. Embora pareça contraditório, inicialmente, é importante recordar que a nãoresposta nesse último grupo levou à utilização posterior de ocitocina.

Os casos em que não se indicou nenhum método de indução foram aqueles internados em trabalho de parto espontâneo ou com indicação de cesárea. Das 11 cesáreas realizadas, 10 foram por iteratividade ( 2 ou 3 cesáreas anteriores).

Em razão dessa pré-seleção do método, com o grupo da ocitocina mais favorável à indução, era de se esperar que o tempo de indução em horas fosse menor para esse grupo. Este estudo mostrou ainda que a dosagem média de misoprostol utilizada foi baixa ( $13 / 4 \mathrm{cp}$ ), diferente do que é amplamente difundido em nosso meio para a indução do parto de feto morto ${ }^{4}$.

Quanto às complicações e intercorrências, 
não houve diferença estatisticamente significativa entre os diferentes métodos de indução e suas associações. Pode-se concluir, portanto, que ocitocina e misoprostol são seguros e eficazes para a indução do parto de feto morto, sendo o misoprostol bastante útil sobretudo em colos imaturos e/ou em gestações com pequena idade gestacional, podendo ser empregado com segurança em baixas doses.

Estes resultados também podem contribuir para o acúmulo de experiência nacional com o uso clínico de misoprostol em obstetrícia, com a finalidade maior de se conseguir a aprovação do uso dessa droga para a indução de parto de gestações com feto vivo, considerando que não se dispõe no Brasil de um agente maturador do colo eficaz para os casos em que a indução do parto se impõe.

\section{SUMMARY}

Although 80 to $90 \%$ of all dead fetuses may be spontaneously eliminated after two to three weeks from death, labor induction has been the mostly used management. The purpose of the current study was to evaluate the results of labor induction for pregnancies with fetal death and gestation age above 20 weeks. It was a descriptive clinical study which was performed at the Hospital e Maternidade Leonor Mendes de Barros in São Paulo, Brazil. One hundred and twenty-two pregnancies with fetal death were evaluated regarding their social and demographic characteristics, causes of fetal death, previous pregnancies history and delivery (induction, route, complications). The statistical procedures used were estimation of mean and standard deviation and $\chi^{2}$. The main causes of fetal death were hypertension and infections. The mostly used drug for labor induction was misoprostol (37.7\%) followed by oxytocin (19.7\%), while $27 \%$ of cases had spontaneous onset of labor. The mean time of induction was 3 hours. The majority of women had vaginal delivery and cesarean section was performed in $9.1 \%$ of them. It is concluded that labor induction for fetal death is safe and efficient, irrespective of the method used. Misoprostol when used in the vagina is specially useful for cases with an unripe cervix because of the modifying effect of the drug on the cervix.

KEY WORDS: Fetal death. Labor. Oxytocin. Prostaglandin.

\section{Referências bibliográficas}

1. Aquino MMA, Cecatti JG. Epidemiologia do óbito fetal. Rev Bras Ginecol Obstet 1998; 20: in press.

2. Behle I. Estudo da contratilidade uterina no parto de feto morto induzido pela injeção intra-ovular de solução de manitol a 20\%. Ginecol Obstet Bras 1979; 2: 525-51.

3. Macau SN. Conduta obstétrica resolutiva na morte fetal anteparto: análise do emprego do misoprostol em baixa dose. São Paulo, 1992. [Tese - Mestrado - Escola Paulista de Medicina\}.

4. Mariani Neto C, Leão EJ, Barreto EMCP, Kenj G, Aquino MMAA, Tuffi VBH. Uso do misoprostol para indução do parto com feto morto. Rev Paul Med 1987; 105: 325-8.

5. Parpinelli MA, Cecatti JG, Ribeiro ST, Besteti Pires HM, Faúndes A. Uso da laminaria no preparo do colo uterino para indução do parto em gestações com óbito fetal. Rev Bras Ginecol Obstet 1996; 18: 693-702.

6. Pritchard JA, Ratnoff OD. Studies of fibrinogen and other hemostatic factors in women with intrauterine death and delayed delivery. Surg Gynecol Obstet 1955; 101: 467-77.

7. Sherman DJ, Frenkel E, Tovbin J, Arieli S, Caspi E, Bukovsky I. Ripening of the unfavorable cervix with extraamniotic catheter balloon: clinical experience and review. Obstet Gynecol Surv 1996; 51: 621-27.

8. Surita FGC. Misoprostol versus laminária no preparo cervical em gestações com óbito fetal. Campinas, 1996. [Dissertação - Mestrado - Universidade Estadual de Campinas].

9. Uldbjerg N, Ulmsten U, Ekman G. The ripening of the human uterine cervix in terms of connective tissue biochemistry. In: Ulmesten U, Ueland K. Clinical Obstetrics and Gynecology; 1983, 26: 14-26.

10. Ulmsten U, Ekman G, Belfrage P, Bygdeman M, Nyberg C. Intracervical versus intravaginal PGE2 for induction of labour at term in patients with an unfavorable cervix. Arch Gynecol 1985; 236: 243-8.

11. Wagatsuma T. Intra-amniotic injection of saline for therapeutic abortion. Am J Obstet Gynecol 1965; 93: 743-5. 